\title{
Ion Trajectory Simulation for Electrode Configurations with Arbitrary Geometries
}

\author{
Guangxiang Wu, R. Graham Cooks, and Zheng Ouyang \\ Department of Chemistry, Purdue University, West Lafayette, Indiana, USA,
}

\author{
Meng Yu and William J. Chappell \\ School of Electrical and Computer Engineering, Purdue University, West Lafayette, Indiana, USA
}

Wolfgang R. Plass

II. Physikalisches Institut, Justus Liebig Universität Giessen, Giessen, Germany

\begin{abstract}
A multi-particle ion trajectory simulation program ITSIM 6.0 is described, which is capable of ion trajectory simulations for electrode configurations with arbitrary geometries. The electrode structures are input from a 3D drawing program AutoCAD and the electric field is calculated using a 3D field solver COMSOL. The program CreatePot acts as interface between the field solver and ITSIM 6.0. It converts the calculated electric field into a field array file readable by ITSIM 6.0 and ion trajectories are calculated by solving Newton's equation using Runge-Kutta integration methods. The accuracy of the field calculation is discussed for the ideal quadrupole ion trap in terms of applied mesh density. Electric fields of several different types of devices with 3D geometry are simulated, including ion transport through an ion optical system as a function of pressure. Ion spatial distributions, including the storage of positively charged ions only and simultaneous storage of positively/negatively charged ions in commercial linear ion traps with various geometries, are investigated using different trapping modes. Inelastic collisions and collision induced dissociation modeled using RRKM theory are studied, with emphasis on the fragmentation of $n$-butylbenzene inside an ideal quadrupole ion trap. The mass spectrum of 1,3-dichlorobenzene is simulated for the rectilinear ion trap device and good agreement is observed between the simulated and the experimental mass spectra. Collisional cooling using helium at different pressures is found to affect mass resolution in the rectilinear ion trap. (J Am Soc Mass Spectrom 2006, 17, 1216-1228) (C) 2006 American Society for Mass Spectrometry
\end{abstract}

$\mathrm{N}$ umerical simulations have been demonstrated to be an effective approach to explore ion trajectories in mass spectrometers of a variety of types including quadrupole ion traps [1], sector instruments [2], and time-of-flight [3] mass spectrometers. Since Dawson performed the first single ion trajectory calculations for a perfect quadrupole field without resonance or collisions [4], simulations have played a particularly important role in the development of ion trap mass spectrometers including Paul ion traps [5] and ion cyclotron resonance $[6,7]$ instruments. With time, more realistic conditions including nonlinear fields [8], buffer gas collisions [9], space charge effects [10], and resonance excitations [11, 12] have been implemented into simulation models. Simulations of the trajectories of multiple ions allow mass spectra to be

Published online June 21, 2006

Address reprint requests to Dr. Z. Ouyang, Department of Chemistry, Purdue University, 560 Oval Drive, West Lafayette, IN 47907, USA, e-mail: ouyang@purdue.edu and Dr. W. R. Plass, II. Physikalisches Institut, Justus Liebig Universität Giessen, 35392, Giessen, Germany, e-mail: Wolfgang.R.Plass@exp2.physik.uni-giessen.de predicted and this tool allows performance characteristics of as-yet-unbuilt instruments to be assessed. In the case of ion trap mass spectrometers, especially smaller instruments and those with simplified electrode structures, optimization of instruments before their construction based on simulations has been highly successful [13-16].

Different ion trajectory simulation programs have been developed and in general the simulations are carried out in two steps, the field solving step and the ion trajectory calculation. Field solving is achieved by using algorithms built into the simulation program or by using separate field solving programs. Ion trajectories can be obtained as analytical solutions to the equations of motion in a particular field or, in most cases, by numerical integration methods. The commercially available ion and electron optics simulation package (SIMION) has been widely applied to the simulations of ion trajectories in custom-designed assemblies of electrodes [17, 18]. The calculation of the static electromagnetic potentials and the ion trajectory are combined in one single package. Users are able to write 
their own algorithms and procedures to implement a variety of simulation conditions like ion initial spatial and velocity distributions, time-varying fields, and ionneutral collisions. The input for these simulations is the geometry of the electrodes and the potentials applied to them. The generality of application is an attractive feature of this program. However, it is not designed for quadrupole ion traps. So many features of these devices, such as ion-neutral collisions, are not supported by default. A program which does focus on ion traps is the integrated system for ion simulation program (ISIS), developed in late 90 s from a series of modules for single ion simulations [19]. It consists of programs for direct integration of the Mathieu equation, the field interpolation method and for quadrupolar resonance [11, 20]. The ion trajectory simulation program (ITSIM), previously known as the ion trap simulation program, has been developed in our laboratory over more than a decade [21-23]. It was originally applied mainly to the study of the behavior of populations of ions inside ion traps under the influence of higher-order fields, buffer gas collisions and space charge. Recently, the capabilities of ITSIM have been extended to allow the simulations of ion trajectories inside electrode assemblies with arbitrary geometries. Another program, the Sx32 simulator [24] includes a user interface, ion generator, and trajectory calculator to simulate ion trajectories for arbitrary devices under the influence of time-varying electric field, collisions, and space charge. The multiphysics simulation system gas and electromagnetic ion optical simulator (GEMIOS) is used to model ion optical systems where electromagnetic forces and gas flow are both important $[25,26]$. This simulation program combines electromagnetic field solutions and fluid dynamic field solutions to simulate ion trajectories.

This paper describes some aspects of the development of ITSIM, the ion trajectory simulation program. The purpose of the development of the previous versions (up to version 5.0) of ITSIM was to provide an educational and research tool for the ion trap mass spectrometry community. Versions 1.0 to 2.5 of ITSIM were developed between 1990 and 1997. The forced Mathieu equation was used to describe the main quadrupole field with secondary effects due to dipolar resonance, elastic collisions, and space charge. The ion trajectory was calculated by solving the forced Mathieu equation using the Euler method. In the applications of these versions of ITSIM in the investigation for ion trap performance, qualitative and semiquantitative information was obtained for various operation modes for the processes of ion injection [27], excitation and ejection $[28,29]$. In 1997, the individual modules for ion generation, ion motion integration, and data analysis were integrated into a single software package, ITSIM 4.0, which is compatible with the Microsoft Windows operating system. Significant effort was put into adding tutorial qualities to make the program heuristic and of value to students and researchers with a variety of backgrounds [22]. The user interface was also of great concern. The embedded stability diagram calculation function allowed the convenient conversions among the operational parameters, such as applied voltages, ion secular frequencies and the Mathieu parameters. Distribution of ITSIM to other research laboratories on request for educational and research purposes started with ITSIM 2.5. The improvement of user interface in ITSIM 4.0 made its application much easier.

The development of ITSIM 5.0 started in 1998. Major changes were made in this version of the program, especially for the core of the simulation model [23]. A matrix field calculation method was implemented for the support of arbitrary electrode geometries with cylindrical symmetry. Different Runge-Kutta methods were used for improved trajectory integration. Models for both elastic and inelastic collisions were added for ion-neutral collisions and collision induced dissociation [30]. Simulation with large numbers of ions, typically 10,000 ions but many more too, is allowed by ITSIM 5.0. Effects due to the fine mechanical features of the ion trap, such as the ejection delay caused by defects in the ion trap configuration [31-33], could be predicted. A study of mass shifts [31-33] in quadrupole ion traps, the development of toroidal trap [15], and cylindrical ion traps $[16,34,35]$ were all greatly facilitated by the use of ITSIM 5.0. A detailed evaluation of these functions in ITSIM was made along with other two simulation tools, ISIS and SIMION [19].

The simulation capabilities of ITSIM 5.0 have been demonstrated to be sufficiently advanced to provide relatively accurate information about ion behavior inside the electric field [23, 36-39]. The effects due to subtle factors in comprehensive processes under complex operating conditions, such as the ejection delay due the combination of the weakened electric field with the presence of the ion-neutral collisions [31-33] and the change of the resolution due to the shift of the resonance AC frequencies [16], can be described using ITSIM 5.0. However, its application was restricted to devices with cylindrical symmetry, such as the Paul [40], toroidal [15], cylindrical [34], and orbital [41] traps, because of the limitation of the field calculation routines to systems of cylindrical symmetry. To apply ITSIM to recently developed linear ion traps [42-44] as well as to use ITSIM to facilitate the development of other mass analyzers and ion optical systems, the capabilities of the ITSIM were extended further. The newest version of this software, ITSIM 6.0, is under development and its new capabilities for simulations in 3D electrical structures are introduced here with a variety of examples.

\section{ITSIM 6.0}

\section{Overview}

As shown schematically in Figure 1, the overall simulation process using ITSIM 6.0 consists of four steps; (1) generating a 3D model of the structure to be used for simulation, (2) solving the electric field inside the struc- 


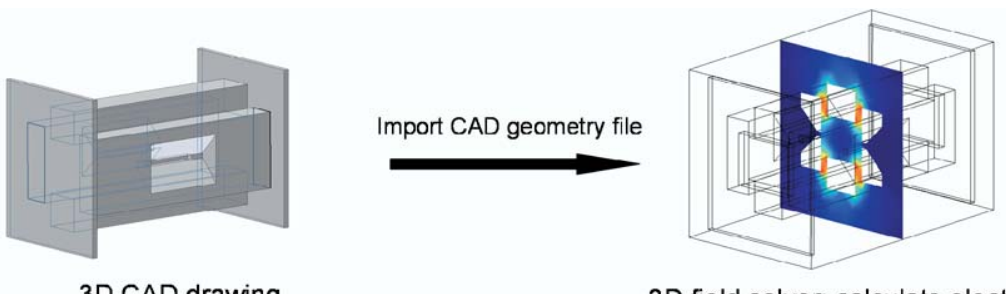

3D CAD drawing

3D field solver: calculate electric field
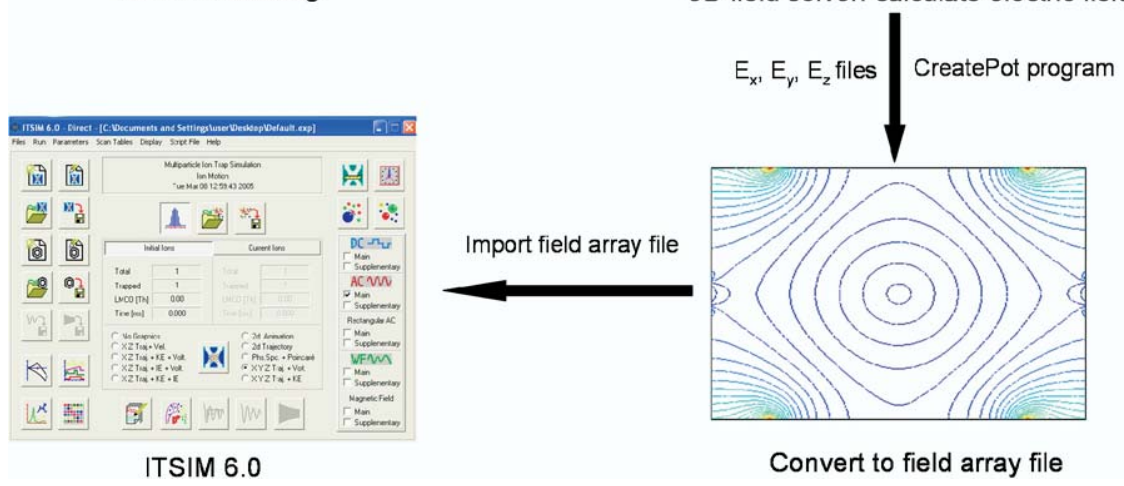

Figure 1. Overall simulation process using ITSIM 6.0.

ture, (3) importing the calculated electric field into the simulation program ITSIM 6.0, and (4) simulating ion motion under specified conditions. The program ITSIM 6.0 uses information on the electric field provided by external field solvers. Development of numerical methods over the past thirty years [45] has resulted in precision 3D numerical solvers and numerous commercial electromagnetic field solvers, particularly those using the finite element method such as COMSOL (COMSOL Inc.; the program was formerly known as FEMLAB) and HFSS (Ansoft Inc.). Typically these field solvers are compatible with commercial mechanical drawing programs and the arbitrary 3D geometric model is generated and the geometry file is then imported into the commercial field solvers. With the assignment of the proper boundary conditions, the electric or magnetic field can be calculated and exported on a specified grid. An interface program of ITSIM 6.0, CreatePot, was developed to convert the electric field solution into a field array file readable by ITSIM 6.0 for ion trajectory simulations.

\section{Ion Motion Simulation}

The newest version of ITSIM, version 6.0, allows the motion of a large number of ions (typically 10,000 but many more are possible) inside an electrical structure to be followed under user-defined experimental conditions. The electric field of the structure is calculated using a field solver program and the field is imported into ITSIM 6.0 in the form of the multipole expansion coefficients or alternatively as a field array file. The default selections allow simulation of conventional 3D traps, 2D linear traps, and ion optical transport systems. Users can also model other electrical structures with arbitrary geometries. The ions are generated in ion groups with user-defined physical properties such as mass, charge, collision cross sections, spatial position, and initial velocity. Collections of ions can be given distributions of values of these parameters. Using scan tables, users can define any temporal sequence and magnitude of applied RF, AC, DC, and arbitrary waveforms voltages, which can be applied to the electrodes. The ion trajectories are calculated by numerically solving Newton's equation of motion using various RungeKutta (fourth, fifth, or eighth order) integration methods with or without step size control. Ion-neutral collisions (elastic, inelastic, and dissociative) and space charge effects can be implemented in the simulations. Depending on the import parameter, the Langevin, hardsphere or a parameterized velocity-dependent collision cross section is used to determine the ion-neutral collision probability. For inelastic collisions, the energy exchange between translational and internal degrees of freedom is described by a transfer probability density that depends on the initial relative kinetic energy, the internal energy of the molecule, and the amount of energy transferred [30]. Ion fragmentation is modeled by using RRKM theory. Multiple data analysis functions can yield plots of electric fields, potentials, and mass spectra, among others. Graphical output of ion motion and velocity, and distributions of these parameters, is also available. An in-depth description of the algorithms used in ITSIM simulations will be published separately.

\section{CreatePot}

CreatePot is an interface program for ITSIM 6.0 used to import field array files. It can convert the output data formats from various field solvers into versions that can be used by the simulation module of ITSIM 6.0. Cur- 
rently, CreatePot is compatible with the 2D solver Poisson Superfish (Los Alamos National Laboratory, Los Alamos, NM) [46], the finite-difference solver SIMION, the 3D finite element solvers COMSOL, and HFSS. When Poisson Superfish is used as the solver, a 2D geometry file is generated in CreatePot and input to ITSIM 6.0. CreatePot automatically calls the solver to calculate the 2D field. The electric potential of the input geometry can be plotted in 2D and the multipole expansion coefficients can be calculated as characteristics of the electric field, a procedure which is particularly useful for ion trap geometry optimization [16]. When using the finite element 3D field solvers, CreatePot takes the electric field output files, typically with electric field values in $\mathrm{x}, \mathrm{y}$, and $\mathrm{z}$ directions and converts them into a field array file readable by ITSIM 6.0. Implementation of an interface between CreatePot and 3D field solvers based on boundary element methods is being developed.

\section{Field Solvers}

The Poisson Superfish program, as a 2D field solver, allows field calculations for geometries with planar or cylindrical symmetry. It covers a volume within specified boundaries with a uniform-sized triangular mesh. A typical mesh density of $1000 \times 1000$ is normally chosen as a compromise between calculated field accuracy and computation time.

For the 3D field solvers, the finite element solvers COMSOL and HFSS have been used with ITSIM 6.0. Both solvers operate by partitioning the structure into tetrahedral mesh to approximate a solution to Maxwell's equations consistent with the boundary conditions of the structure. COMSOL, with the electromagnetic module, allows the calculation of quasi-static solutions to Laplace's equation in which the electric field is extracted from the calculated voltage at each node of the tetrahedral. HFSS is more suitable in complex analyses where high-frequency simulations are required. The tetrahedral mesh is adaptively refined, which is proportional to the electric field gradient, and the electric field vectors are solved directly. The selection of the finite element solver is dependent on the size of the structure, the frequency of the trapping RF, etc. In the case of ion traps, which are operated in a low-frequency region $(\sim 1 \mathrm{MHz} R \mathrm{R}$ and static DC), COMSOL usually provides a sufficiently accurate field with a shorter computation time than HFSS.

For arbitrary 3D geometries, COMSOL normally uses a repetitive tetrahedral mesh element to define the region, and the field is calculated at each node of the tetrahedral by solving partial differential equations. In this study, the 3D drawing of the electric components was first generated using a CAD program at a 1:1 scale, and the geometric center of the arbitrary volume was chosen as the Cartesian origin. The geometry file was then imported into COMSOL. The "electrostatics" mode was chosen for all electric field calculations. To set the physical boundary conditions, the electric components were either assigned "electric potential" with normalized $\pm 1 \mathrm{~V}$ or treated as "ground" with $0 \mathrm{~V}$. During the simulation, the actual field strength at an arbitrary position is obtained by a superposition of the field solutions for each electrode scaled by the applied voltages.

The accuracy of the solved field is usually dependent on the mesh density, which is always limited by the available computational power. Without specification, the default mesh function of finite element field solvers automatically partitions more mesh elements around regions with sharp edges or strong curvature. To reduce error accumulation during numerical calculations, a smaller mesh size, corresponding to a higher mesh density, can be specified in the region where the ion trajectory is calculated. This is discussed further in the following section. Compromises are often made between the accuracy of calculated field and the availability of the computer memory. To reduce the computation time and memory requirement when using COMSOL, an iterative linear solver that is suitable for solving unsymmetrical problems was selected for all the simulations, and an algebraic multigrid preconditioner was chosen that works best for scalar elliptic partial differential equations such as Laplace's equation and Poisson's equation. After completion of the 3D field calculations, the electric field data in the $x, y$, and $z$ directions were exported as a text file using a regular grid and the grid size was chosen to cover 100 data points in each direction. To get the best simulation accuracy with the minimum requirement for computing power, field conversion using nonuniform grid is highly desirable. This will require further development of CreatePot and ITSIM in the future.

\section{Field Accuracy}

The accuracy of the electric field calculation is critical for the simulation. The field accuracy that can be achieved is directly related to mesh density, which is in practice limited by the computer memory size. To optimize conditions for the simulation at a given computing power, several methods can be used to reduce the overall mesh size during the field solving. Variable mesh size can be used and higher mesh density can be set for the region where ion trajectories are to be calculated. This is shown in Figure 2a, the 3D tetrahedral mesh plot from COMSOL 3.0a, which shows the field in the central part of a Paul trap solved with a refined smaller mesh size. If the structure has cylindrical symmetry as does an ideal Paul trap (Figure 2b), or planar symmetry as does the ideal quadrupole mass filter (Figure 2c), only a fraction of the field volume, the 2D cylindrical symmetric subspace for the Paul trap and one-eighth of the whole 3D volume for the quadrupole mass filter needs to be solved and the entire field solution can be derived based on symmetry. The field with cylindrical symmetry is usually most computa- 
(a)

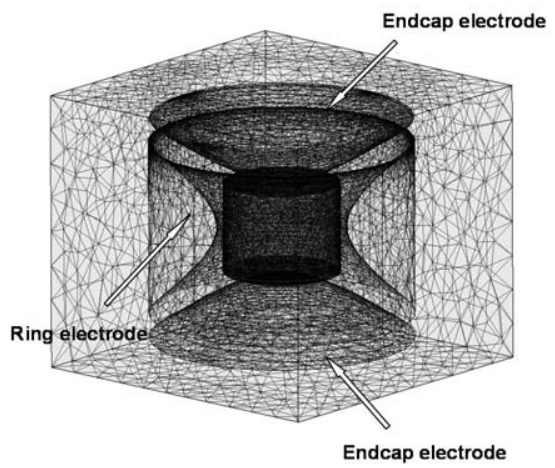

(b)

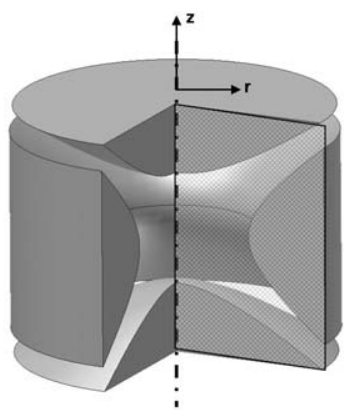

(c)

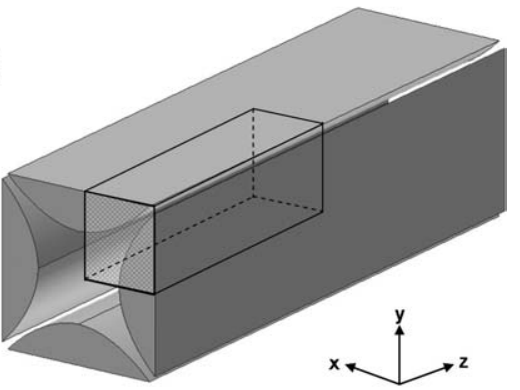

Figure 2. (a) $3 \mathrm{D}$ tetrahedral mesh plot for the Paul trap with ideal geometry $\left(\mathrm{r}_{0}=10 \mathrm{~mm}, \mathrm{z}_{0}=7.07\right.$ $\mathrm{mm}$ ) but truncated electrodes; (b) 2D planar mesh for ideal Paul trap which has cylindrical symmetry along the $\mathrm{z}$ axis; (c) $1 / 8$ volume mesh for the ideal but truncated quadrupole mass filter which only has planar symmetry along the $x-y, y-z$, and $x-z$ planes.

tionally efficient, since only a 2D field needs to be solved.

The required field accuracy varies significantly depending on the simulation task specified. To illustrate the effect of the field accuracy on the simulation results, comparisons can be made between simulations using fields solved with different mesh densities. The field inside an ideal geometry Paul trap, $\mathrm{r}_{0}=10 \mathrm{~mm}, \mathrm{z}_{0}=$ $7.07 \mathrm{~mm}$, no endcap holes and the electrode truncation radius 2.5 times $\mathrm{r}_{0}$, was calculated using COMSOL 3.0a utilizing the different methods summarized in Figure $2 a$ and $b$. When the 3D field is solved entirely using COMSOL 3.0a with a desktop computer of $3.0 \mathrm{GHz}$ CPU and 3.0 GB RAM, an allowable maximum average mesh density about $10^{3} \mathrm{~cm}^{-3}$ was used and the maximum mesh element size was $0.8 \mathrm{~mm}$. When the 2D planar field was solved, a much higher average mesh density, about $10^{4} \mathrm{~cm}^{-2}$, could be used and the maximum mesh element size was only $0.1 \mathrm{~mm}$. The solved fields were compared with that derived from analytical solution. Recall the electric potential for the Paul trap with ideal geometry in the Cartesian coordinates:

$$
\Phi=\frac{\Phi_{0}}{2 r_{0}{ }^{2}}\left(\alpha x^{2}+\beta y^{2}+\lambda z^{2}\right)
$$

where $\Phi_{0}$ is the potential applied between the ring electrode and the pair endcaps, $r_{0}$ is the inscribed radius of the ring electrode. The Laplace condition imposes the constraint:

$$
\alpha+\beta+\lambda=0
$$

so for the 3D configuration, this allows:

$$
\alpha=\beta=1, \lambda=-2
$$

The electric field strengths in the $x, y$, and $z$ directions are then given by:

$$
E_{x}=-\frac{\Phi_{0}}{r_{0}^{2}} x, E_{y}=-\frac{\Phi_{0}}{r_{0}^{2}} y, E_{z}=\frac{2 \Phi_{0}}{r_{0}^{2}} z
$$

Applying the initial boundary conditions $\Phi_{0}=1 \mathrm{~V}$ at $\mathrm{r}_{0}=10 \mathrm{~mm}$, eq 4 can be simplified as:

$$
\begin{aligned}
& E_{x}=-0.01\left(\mathrm{~V} / \mathrm{mm}^{2}\right) * x, E_{y}=-0.01\left(\mathrm{~V} / \mathrm{mm}^{2}\right) * y, \\
& E_{z}=0.02\left(\mathrm{~V} / \mathrm{mm}^{2}\right) * z
\end{aligned}
$$

The calculated electric fields $E_{x}, E_{y}$, and $E_{z}$ from COMSOL 3.0a using the same initial boundary conditions are all within $2 \%$ deviation from the analytical solution for lower average mesh density (order of $10^{3}$ $\mathrm{cm}^{-3}$ ), but within $0.35 \%$ for the higher average mesh density (order of $10^{4} \mathrm{~cm}^{-2}$ ). In both cases, and as expected, the largest deviation between the calculated electric field and the analytical solution occurs in the areas closest to the electrode boundaries. The solved fields were imported to ITSIM 6.0 and the ion trajectories were simulated for a single ion of $m / z 100$. The ion was defined as having an initial position $x=y=3 \mathrm{~mm}$, $\mathrm{z}=4 \mathrm{~mm}$, and zero initial velocity. The fourth order Runge-Kutta integration method was used with an integration step of 10 ns. A constant RF voltage of 1.1 $\mathrm{MHz}$ and $450 \mathrm{~V}_{0-p}$ was applied to the ring electrode 


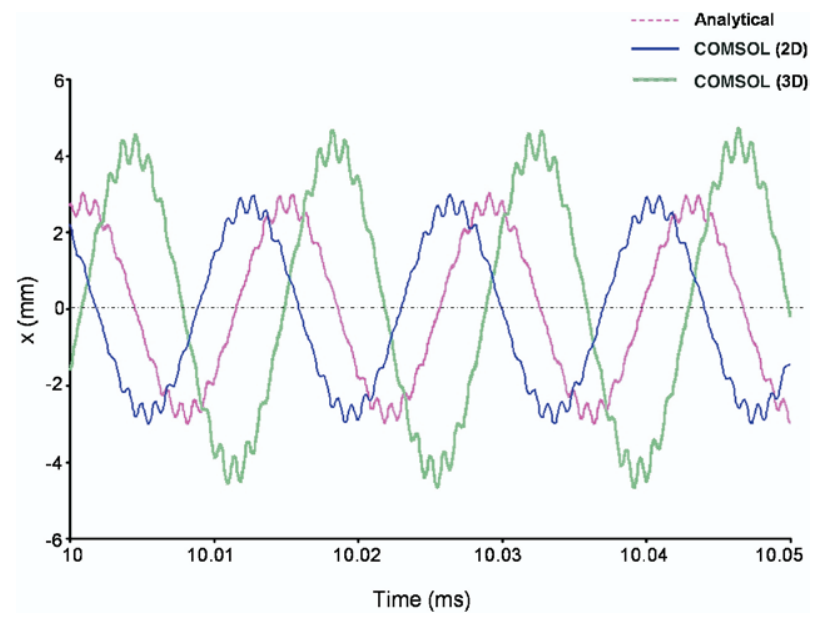

Figure 3. Ion trajectories calculated in the $x$ direction for an ideal Paul trap as a function of time, 10 to $10.05 \mathrm{~ms}$ after the start of the simulation. The ion trajectories shown are obtained using an analytical solution (Mathieu equation) and numerical solutions (COMSOL 3.0a with field solved using two different mesh densities).

with the two endcap electrodes grounded. Collision and space charge effects were not included in this simulation. The simulated trajectories are compared with that derived analytically using Mathieu equation, as shown in Figure 3. After $10 \mathrm{~ms}$, a phase shift of 0.004 ms was found for the trajectory simulated with a lower average mesh density (order of $10^{3} \mathrm{~cm}^{-3}$ ) and a deviation in maximum amplitude was also observed. The error in the simulated result was mainly due to inaccuracy in field solving, not in the integration method. The accuracy of the integration method has been characterized previously using multipole expansions to describe the field, and in a representative case a shift from the analytical solution of only $0.0005 \mathrm{~ms}$ in $10 \mathrm{~ms}$ was found for given conditions [23]. With a higher average mesh density (order of $10^{4} \mathrm{~cm}^{-2}$ ), the phase shift was decreased to half, $0.002 \mathrm{~ms}$, and an amplitude was identical to the theoretical one.

\section{Model Simulations}

The capabilities of the newest version of ITSIM 6.0 were demonstrated by simulations of ion motion in a variety of electrical structures. The highest mesh density allowable by the computer memory was chosen for field solving using COMSOL 3.0a. The results of the simulations are described in the sections which follow. For convenience, the set of simulations is listed here. (1) Ion focusing behavior was studied for a system of static lens and a system of square quadrupoles, in each case as a function of operating pressure. (2) Mass spectra of 1,3-dichlorobenzene were simulated using the boundary ejection version of the mass-selective instability operating mode (increasing the amplitude of the RF trapping voltage) for the rectilinear ion trap (RIT) [44]. (3) Collisional cooling with different helium buffer gas pressures was studied for its effects on mass resolution. (4) Inelastic collisions leading to collision induced dissociation (CID) were also studied for the fragmentation pathway, $m / z 134$ to 92 , of $n$-butylbenzene within the ideal Paul trap. (5) Ion distributions were investigated for two types of linear ion traps operated in different trapping modes.

Except as otherwise indicated, the simulations were performed using an ensemble of ions Gaussian distributed from the center of the electrical structure with 0.3 $\mathrm{mm}$ standard deviation in the $\mathrm{x}, \mathrm{y}$, and $\mathrm{z}$ directions. All ions were assigned the hardsphere cross section of 50 $\AA^{2}$ and a parameterized velocity-dependent collision cross section is used to determine the ion-neutral collision probability. For CID, the internal energy-transfer between translational and internal degrees of freedom was modeled by a transfer probability density model followed by the fragmentation using RRKM theory [30]. The fourth order Runge-Kutta integration method was used to calculate ion trajectories using a $10 \mathrm{~ns}$ step size. No space charge effects were included in the simulation.

\section{Ion Focusing Using Static Electric Fringing Fields}

Ion focusing using fringing fields is commonly used in ion optical systems, which involve various components of an ion optical system. The quality of the focusing is highly dependent on the configuration of the lens elements, such as the diameter of the holes, the inter-electrode distance, the thickness of, and the voltages applied to, the individual lens elements. Simulation is often used in the design process to seek optimized conditions for the lens. A three element lens was used for the simulation shown in Figure 4a. Each of the lens electrodes is $2.0 \mathrm{~mm}$ in thickness and has a centered hole with a radius of $5.0 \mathrm{~mm}$. The distance between adjacent electrodes is $1.54 \mathrm{~mm}$. DC voltages of, $-90,-20$, and $-70 \mathrm{~V}$, were applied to the three electrodes. An ensemble of ions with $\mathrm{m} / z 100$ passing through the lens was simulated. The ions were defined by a uniform initial distribution in both $x$ and $y$ directions within the region from -0.2 to $+0.2 \mathrm{~mm}$. In the $\mathrm{z}$ direction, the ions started from $-4.54 \mathrm{~mm}$ and had an initial kinetic energy of 16.5 $\mathrm{eV}$. Elastic collisions between ions and helium buffer gas, at pressures of 0.01 and 5 mTorr, were also included. The ion trajectory in $x-z$ plane using this lens system is shown in Figure $4 \mathrm{~b}$. At lower pressure condition (0.01 mTorr), focusing of the ions is achieved clearly by a combination of ion acceleration and deceleration, as is usual for fringing field lenses. However, in the case with higher pressure (5 mTorr) and thus harsher collision conditions, ions are more scattered after deceleration and could not be focused to the single focal point along the $\mathrm{z}$ axis. 

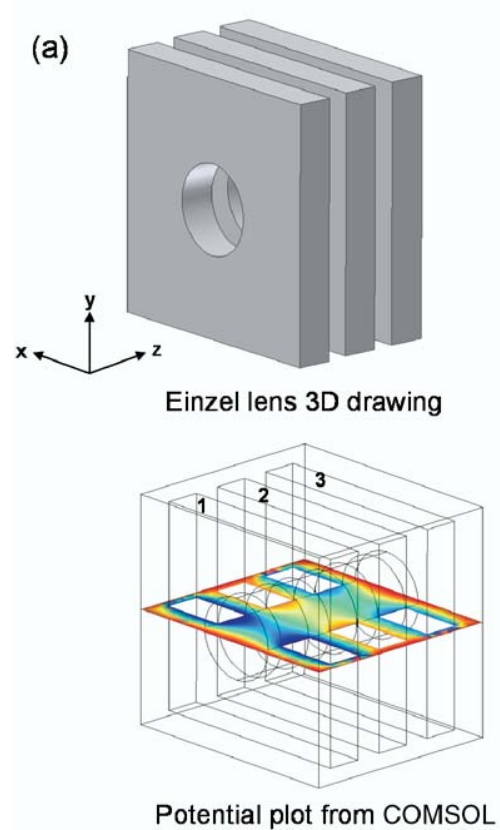

(b)
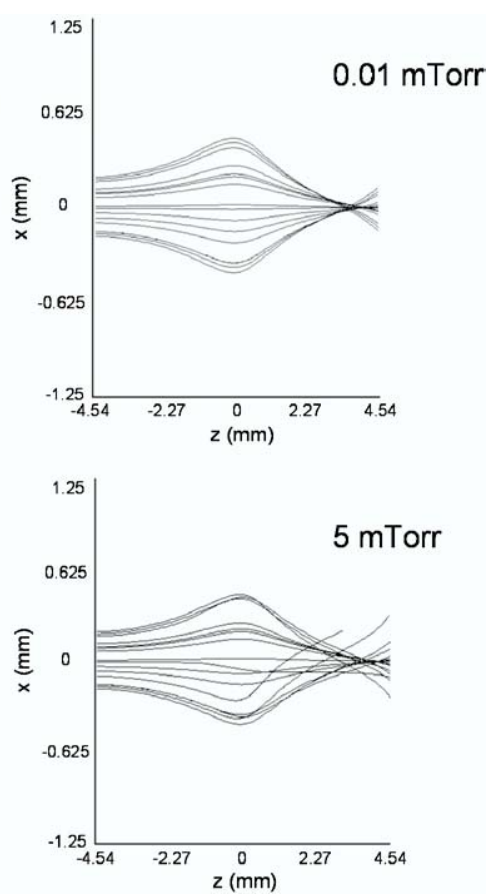

Figure 4. (a) 3D view for a static lens and the potential plot from COMSOL 3.0a, which shows applied DC voltages, $-90 \mathrm{~V}$ (lens 1), $-20 \mathrm{~V}$ (lens 2), and $-70 \mathrm{~V}$ (lens 3 ) and the calculated 2D potential. (b) Ion focusing in $\mathrm{x}-\mathrm{z}$ plane of $\mathrm{m} / \mathrm{z} 100$ ions of $16.5 \mathrm{eV}$ initial kinetic energy in presence of helium buffer gas at 0.01 and 5 mTorr.

\section{Ion Transfer Using RF Ion Guides}

Besides static lens systems, ion guides based on focusing with RF fields are widely used in mass spectrometry, especially when passing ions through regions of different pressure. With different collision effects at various pressures, different multipole ion guides have been designed, and their working conditions are normally optimized to obtain the highest ion transfer efficiency over a wide range of mass-tocharge ratios. Figure 5a shows a square quadrupole ion guide, with two-phase RF voltages $\left(180^{\circ}\right.$ phase difference) applied between the two pairs of rectangular-shaped electrodes. The ions are confined in the $\mathrm{x}$-y plane by the RF field. Each electrode is $4.9 \mathrm{~mm}$ in width, $6.22 \mathrm{~mm}$ in height, and the half distance between each pair electrodes is $2.78 \mathrm{~mm}$. The total length of the electrodes in the $\mathrm{z}$ direction is $20 \mathrm{~mm}$. In the simulations, an ensemble of ions with $\mathrm{m} / \mathrm{z} 200$ was defined with a uniform initial distribution in both the $x$ and $y$ directions and in a region from -0.2 to +0.2 $\mathrm{mm}$. In the $\mathrm{z}$ direction, the ions started from $-12 \mathrm{~mm}$ and had an initial kinetic energy of $14 \mathrm{eV}$. An RF of $1.0 \mathrm{MHz}, 100 \mathrm{~V}_{0-p}$ was applied to the two pairs electrodes. Ions were simulated under two different helium buffer gas pressures: 1 and 100 mTorr. The oscillation of the ion beam size, which is typically observed for quadrupole ion guides [47], is shown in simulation at low-pressure (Figure $5 b$ ). With the increase of the buffer gas pressure, the ions motion becomes more random with loss of the regular focusing and defocusing pattern along the ion optical axis.

\section{MS Performance of a Rectilinear Ion Trap}

The analytical performance of an ion trap mass analyzer is dependent on many factors, including the electric field, ion ejection methodology and conditions, and pressure [5]. The geometry of the trap, the frequency of the RF, the amplitude and frequency of the supplementary AC used for resonance ejection, and the molecular weight of the buffer gas all play important roles in the mass resolution of a trap. Optimization of the performance is usually an iterative process in which each of the parameters is adjusted successively. Simulations can provide guidance in the design and optimum operation of ion traps. Applying the same scan functions used in the experiment, ITSIM 6.0 can simulate mass spectra and thus provide direct comparisons with experimental results. The rectilinear ion trap (RIT) [44] is a new type of linear ion trap with an exceptionally simple geometry. It consists of $x$ and $y$ pairs of rectangular-shaped electrodes and flat pair of $\mathrm{z}$ electrodes, as shown in Figure 6a. As with any ion trap, there are different operation modes for the RIT. For instance, (1) either single-phase or two-phase RF can be applied to the $x$ and $y$ pair electrodes to confine ions in the $x-y$ plane, (2) either DC or AC signals can be applied to the $\mathrm{z}$ pair electrodes to confine the trapped ions along the $\mathrm{z}$ axis, (3) ions can be ejected either radially through the slits in the $x$ pair of electrodes or axially through the holes in the $\mathrm{z}$ pair of electrodes [48, 49]. For the modeled RIT, the half distance between the $x$ pair electrodes is $5.0 \mathrm{~mm}$, the half distance between y pair electrodes is $4.0 \mathrm{~mm}$, the length of $\mathrm{x}$ and $\mathrm{y}$ pairs of electrodes is $40.0 \mathrm{~mm}$. The $\mathrm{z}$ pair electrodes are spaced $1.5 \mathrm{~mm}$ distant away from the ends 
(a)

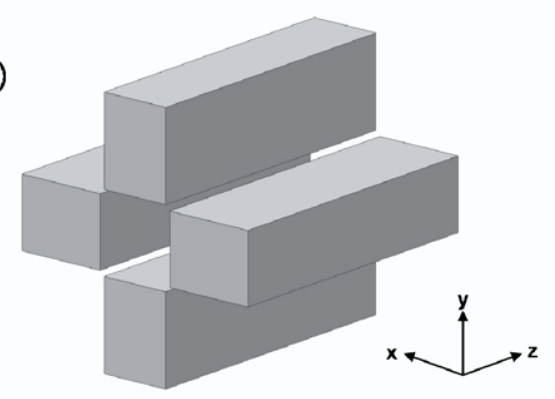

Square quadrupole 3D drawing

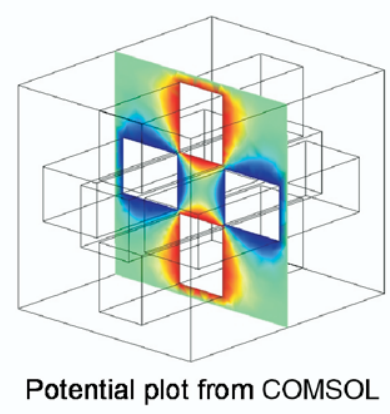

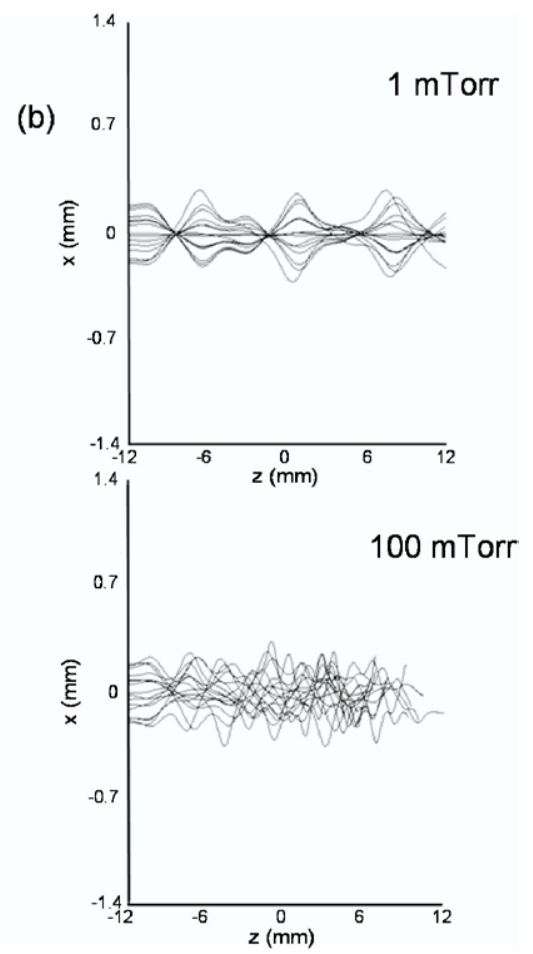

Figure 5. (a) $3 \mathrm{D}$ view of a square quadrupole and the potential plot from COMSOL 3.0a, which shows the two-phase RF applied to the two pairs of electrodes. (b) Ion motion in x-z plane. Ions of $\mathrm{m} / \mathrm{z}$ 200 move through the square quadrupole with initial kinetic energy $14 \mathrm{eV}$ while an RF of $1.0 \mathrm{MHz}$, $100 \mathrm{~V}_{0-p}$, is applied to the two pairs of electrodes. Two different helium buffer gas pressures, 1 and 100 mTorr, were studied.

of the $\mathrm{x}$ and $\mathrm{y}$ electrodes and had no holes in this case. A single-phase RF was applied to the y pair electrodes leaving $x$ pair electrodes grounded and one DC voltage was applied to the $\mathrm{z}$ pair electrodes.

The mass spectrum of 1,3-dichlorobenzene created by boundary ejection through the slits in the $x$ electrodes was simulated. Ensembles of ions with mass-to-charge ratios and abundances which correspond to its standard EI spectrum were generated in ITSIM 6.0. The simulation conditions were chosen based on the experimental conditions. The initial RF applied to the y pair of electrodes was $1.1 \mathrm{MHz}, 200 \mathrm{~V}_{0-p}$ and the RF amplitude was increased at a rate of $67.8 \mathrm{~V} / \mathrm{ms}$. A DC voltage of $200 \mathrm{~V}$ was applied to the $\mathrm{z}$ pair electrodes. The helium buffer gas pressure was 0.8 mTorr. The good agreement between the experimental and simulated mass spectra of 1,3-dichlorobenzene is evident from Figure $6 \mathrm{~b}$. Note that the comparison does not include a consideration of inelastic collisions and energytransfer since the fragmentation is an input in the simulations. In Figure $6 c$, collisional cooling using helium at various pressures is studied for its effects on mass resolution. The only difference between the simulations of Figure $6 c$ and $b$ is that for Figure $6 b$, all the ions were initially Gaussian distributed from the center of the RIT ( $x$ $=\mathrm{y}=\mathrm{z}=0$ ) with $0.3 \mathrm{~mm}$ standard deviation in the $\mathrm{x}, \mathrm{y}$, and $z$ directions, while for Figure $6 c$ all the ions started initially with uniform distribution along $x(0 \pm 5.0 \mathrm{~mm}), y$ $(0 \pm 4.0 \mathrm{~mm})$ and $\mathrm{z}(0 \pm 20 \mathrm{~mm})$ directions. Three different helium buffer gas pressures were chosen: $8,0.8$, and 0.08
mTorr. The collisional cooling at 0.8 mTorr gives the best mass resolution in the simulated mass spectrum in terms of the separation of the isotopic peaks $m / z 146,148$, and 150 . Both higher pressure ( $8 \mathrm{mTorr}$ ) and lower pressure (0.08 mTorr) degrade the mass resolution. This can be explained as the result of the increased collision frequency at higher buffer gas pressure ( 8 mTorr); the scattering caused by harsh collision will alter the phase relation between the ion motion and RF field. Some ions are ejected earlier or later than the majority of ions of the same mass-to-charge ratio. As the mass spectrum is acquired as a function of time, any difference in ejection time will degrade the mass resolution. On the other hand, with the decreased collision frequency at lower buffer gas pressure (0.08 mTorr), ions have less chance to be collisional cooled to the central volume of the trap. Thus, ions are more easily ejected or never acquire stable trajectories and both signal intensity and mass resolution will decrease dramatically.

\section{Collision Induced Dissociation}

Besides the capability of simulating the effects of elastic collisions, ITSIM 6.0 can also be used for the study of the processes involving inelastic collisions. A typical example is the simulation of CID, where the internal energy of a analyte parent ion is increased due to the collisions between the ion and the neutral buffer gas molecule and the parent ion subsequently fragments as a result. For a 


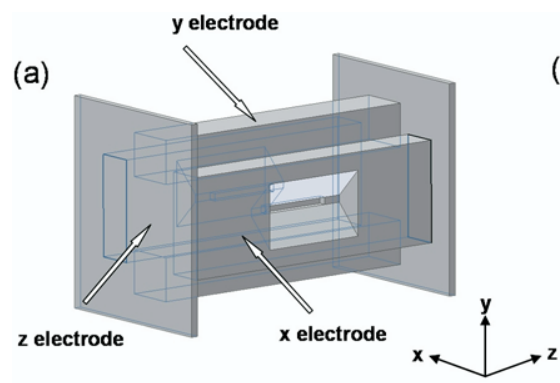

Rectilinear ion trap 3D drawing

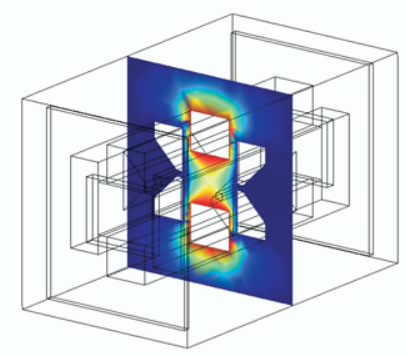

Potential plot from COMSOL
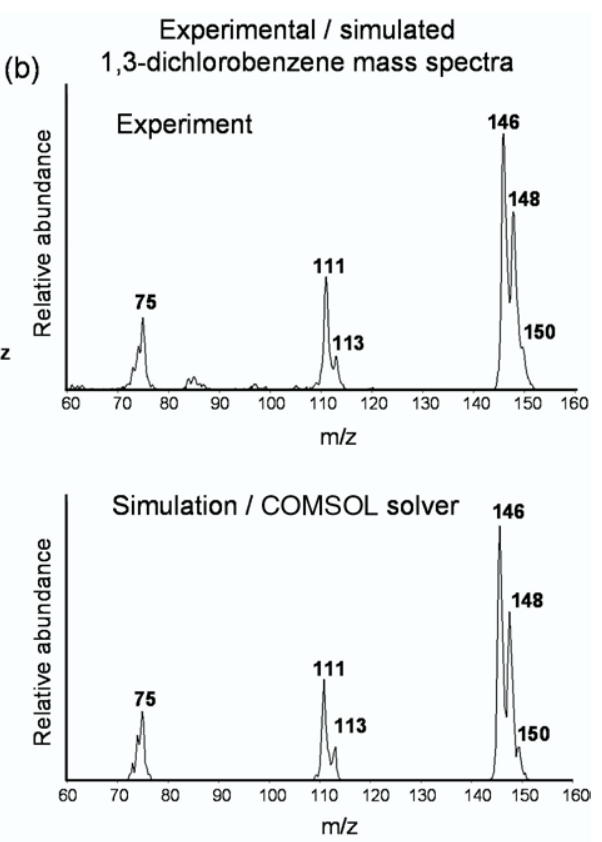

(c)

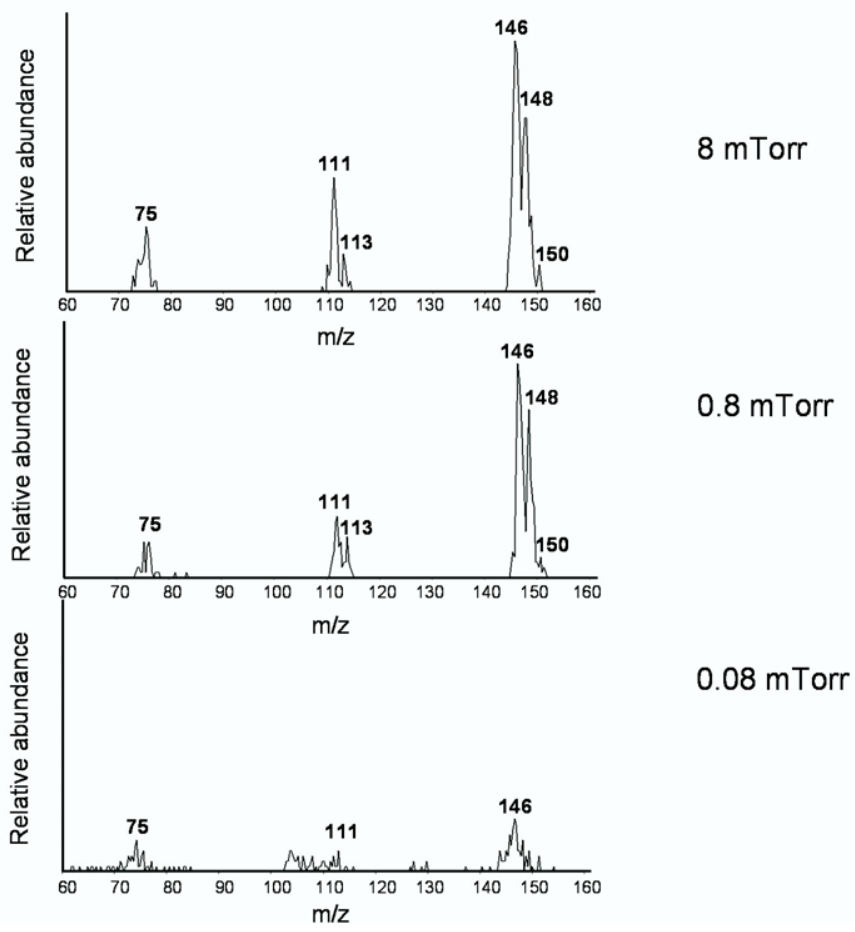

Figure 6. (a) $3 \mathrm{D}$ view of the rectilinear ion trap (RIT) and the potential plot from COMSOL 3.0a showing the field when one-phase RF is applied to the $y$ pair electrodes while the $x$ and $z$ pair electrodes are grounded. (b) Experimental and simulated internal EI mass spectrum for 1,3-dichlorobenzene using boundary ejection. The initial RF applied to the y electrodes was $1.1 \mathrm{MHz}, 200 \mathrm{~V}_{0-p}$, and the RF amplitude was scanned at $67.8 \mathrm{~V} / \mathrm{ms}$. The DC voltage applied to the $\mathrm{z}$ pair electrodes was $200 \mathrm{~V}$. The helium buffer gas pressure was 0.8 mTorr. (c) Simulated mass spectra of chemical compound 1,3-dichlorobenzene using boundary ejection with initial uniform spatial distribution along the $\mathrm{x}, \mathrm{y}$ and $\mathrm{z}$ directions. Three different helium buffer gas pressures were chosen: $8,0.8$, and 0.08 mTorr.

specific chemical system, users are able to input the physical and chemical properties of the system and to vary the parameters for the collision process. Typical variable properties include fragmentation pathways, critical energies, vibrational and rotational frequencies, etc.
[50]. The initial internal energy distribution and energy transferring efficiency can be varied in the simulation of the collision process. An example is given here for the simulation of the CID process for the well-studied molecule $n$-butylbenzene. The energy exchange between trans- 


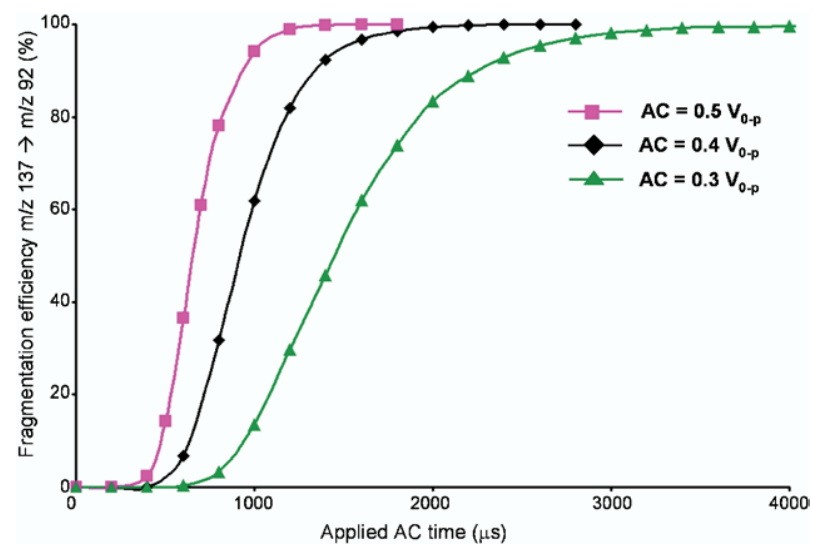

Figure 7. Fragmentation efficiency of $n$-butylbenzene molecular ions, pathway $m / z 134$ to 92 , as a function of applied AC amplitude in ideal Paul trap $\left(\mathrm{r}_{0}=10 \mathrm{~mm}, \mathrm{z}_{0}=7.07 \mathrm{~mm}\right)$. The RF applied to the ring electrode was $371.54 \mathrm{~V}, 1.0 \mathrm{MHz}$, and the frequency of the AC applied in the dipolar mode was $97.18 \mathrm{kHz}$ and three different amplitudes: $0.3,0.4$, and $0.5 \mathrm{~V}_{0-p}$ were explored.

lational and internal degrees of freedom was modeled using a transfer probability density model. The probability density for collisions that lead to excitation is assumed to decrease exponentially with the amount of transferred energy, while for de-excitation, it is obtained from microscopic reversibility [30]. The study of the CID process in the ion trap helps in the understanding of the cooling and excitation process and illustrates the behavior of a particular chemical system in a particular device. Shown in Figure 7 is the fragmentation efficiency of $n$-butylbenzene with variation in the amplitude of the applied AC using a Paul trap of ideal geometry $\left(\mathrm{r}_{0}=10 \mathrm{~mm}, \mathrm{z}_{0}=7.07 \mathrm{~mm}\right)$. For the simulation, an ensemble of 10,000 $n$-butylbenzene ions of $\mathrm{m} / \mathrm{z} 134$ was defined in space at the center of the trap. The standard deviation in the $\mathrm{x}$ and $\mathrm{y}$ directions was $0.46 \mathrm{~mm}$ and it was 0.36 in the $\mathrm{z}$ direction. All these ions had the same hardsphere collision cross section $\left(73.3 \AA^{2}\right)$ and the same internal energy $(0.2 \mathrm{eV})$. The input vibrational frequencies of $n$-butylbenzene were taken from Baer et al. [51] and the critical energies for two dissociation channels, $m / z 134$ to 92 and $m / z 134$ to 91 , were taken to be $0.99 \mathrm{eV}$ and $1.61 \mathrm{eV}$, respectively. The energy transferring efficiency was set as 0.1. Possible consecutive dissociation from $m / z 92$ ion to $m / z 91$ was neglected. The RF applied to the ring electrode was $371.54 \mathrm{~V}_{0-p}, 1.0 \mathrm{MHz}$, which corresponds to Mathieu parameter $\mathrm{q}_{\mathrm{z}}=0.27$. The frequency of the resonant $\mathrm{AC}$ applied in the dipolar mode was 97.18 $\mathrm{kHz}$ which matches with the secular frequency of $m / z 134$ ion. Three different applied AC voltages were investigated $0.3,0.4$, and $0.5 \mathrm{~V}_{0-p}$. The total simulation time was $5 \mathrm{~ms}$ and ion-electrode collisions were not considered. During the collision process, the helium buffer gas pressure was chosen to be 2 mTorr and both elastic and inelastic collisions were considered, a change in the internal energy being applied whenever an inelastic collision occurred. A "detailed balance" general model [50], which was applied to the equilibrium system where the rate of each process is exactly balanced by the rate of the reverse process, was used to define the internal energy-transfer and the Rice-Rampsberger-Kassel-Marcus (RRKM) theory was used to describe the unimolecular dissociation of the population of $m / z 134$ ions. In Figure 7, it can be seen that for each amplitude of the resonant AC voltage, the fragmentation of $n$-butylbenzene only occurs by the pathway $\mathrm{m} / \mathrm{z} 134$ to 92 due to the lower critical energy for fragmentation. The fragmentation efficiency of ion $\mathrm{m} / \mathrm{z} 134$ to 92 increases with the time of AC application. The larger the applied voltage, the less the time needed to reach $100 \%$ fragmentation.

\section{Ion Distribution Inside a Linear Ion Trap}

Linear ion traps have recently seen rapid development due in part to their increased ion trapping capacities. The manipulation of the ions in the linear trap has been studied with the help of simulations [24, 42, 43]. Whether the ions are ejected orthogonally or axially, the increased ion trapping capacity could be beneficial to increase the detected ion signal and to decrease detection limits. However, the possibility of space charge effects that will degrade resolution must be considered. Thus, elucidation of the stored ion cloud distribution is critical to optimize the performance of linear ion traps. Figure 8a models the commercial Thermo LTQ linear ion trap, which consists of three sections of hyperbolic rods and one pair of flat endplates. The hyperbolic rods have an inscribed radius of $4.75 \mathrm{~mm}$ (x axis) and $4.0 \mathrm{~mm}$ (y axis). The lengths of the three section rods are $12 \mathrm{~mm}$, $37 \mathrm{~mm}$, and $12 \mathrm{~mm}$, respectively. The gap between each section is $1.0 \mathrm{~mm}$. A centered slit with $30 \mathrm{~mm}$ length and $0.25 \mathrm{~mm}$ width is cut through each of the middle section $x$ rod pairs. The endplates have a $3.0 \mathrm{~mm}$ diameter hole in the center and are spaced $1.5 \mathrm{~mm}$ away from the ends of the axial rods. During ion trapping, a DC voltage is applied to the three sections of rods in such a way that the DC offset between the rods in the central section and the other two forms a DC potential well, which confines the ions along the $\mathrm{z}$ axis. In the simulations, 10,000 ions of $\mathrm{m} / \mathrm{z} 100$ were placed in the center of the linear trap. Two-phase RF, $1.0 \mathrm{MHz}$ and $100 \mathrm{~V}_{0-p}$, was applied to the central section rods. The other two rod sections were each applied with the same DC voltage ( 1 or $10 \mathrm{~V})$ and the two endplates were held at a constant $6 \mathrm{~V}$ DC. The simulation time was $10 \mathrm{~ms}$ and the helium buffer gas pressure was $1 \mathrm{mTorr}$. Shown in Figure $8 \mathrm{~b}$ are the ion distributions in $\mathrm{x}-\mathrm{z}$ plane when either a 1 or a $10 \mathrm{~V}$ DC potential well is applied between the central and other two sections of rods. The depth of color from dark to gray corresponds to the decreasing statistical possibility of detecting ions in a specific region at a certain time. Clearly using a $10 \mathrm{~V}$ DC potential well, ions are confined in a small region, 20 $\mathrm{mm}$ long and $0.6 \mathrm{~mm}$ wide. For comparison, using a 1 V DC potential well, the ions are spread out in a larger region, $25 \mathrm{~mm}$ long and $0.6 \mathrm{~mm}$ wide. 

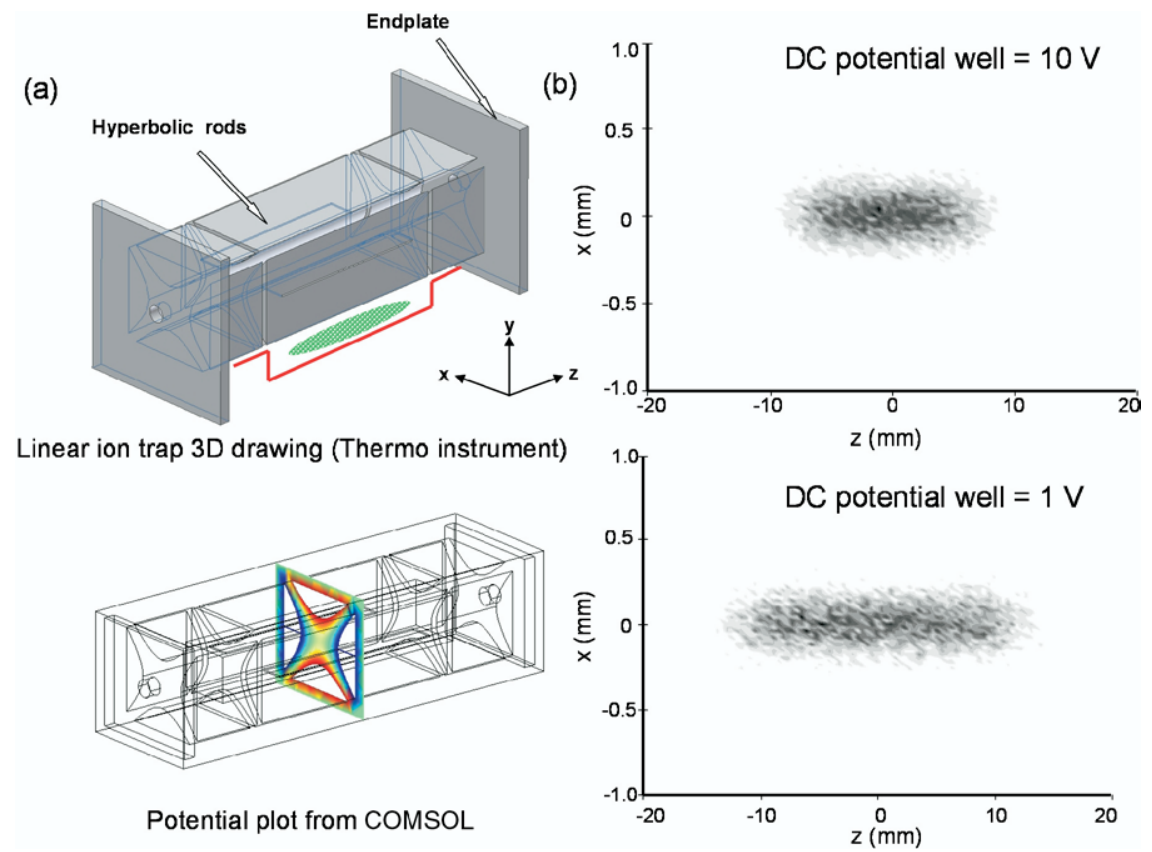

Potential plot from COMSOL

Figure 8. (a) 3D view for the Thermo linear ion trap and potential plot from COMSOL 3.0a, which shows the field resulting from application of the two-phase RF to the two pairs of electrodes comprising the middle rod section. (b) Ion distribution for $m / z 100$ ions in $x-z$ plane with either 1 or $10 \mathrm{~V}$ DC potential were applied between the central and two end rod sections. Two-phase RF was applied to the central section of rods with a frequency $1.0 \mathrm{MHz}$ and an amplitude of $100 \mathrm{~V}$; the two end sections of rods were applied with the same DC voltage, 1 or $10 \mathrm{~V}$, and the two endplates were kept constant at $6 \mathrm{~V}$ DC. The helium buffer gas pressure was $1 \mathrm{mTorr}$. The color from dark and gray corresponds to the decreasing statistic possibilities to detect ions in a specific region at a certain time.

\section{Mutual Storage of Positive and Negative Ions}

Ion-ion reactions have been successfully applied in the analysis of proteins and peptides using mass spectrometry. One application of ion-ion reactions is simplification of ESI spectra, which is achieved by reducing the charge states of the ionic species. The recent development of electron-transfer dissociation $[52,53]$ provides a powerful tool for top-down proteomics, especially when used in conjunction with ion-ion reactions. Ionion reactions were initially carried out in 3D ion traps using pseudopotential wells to trap both positive and negative charged ions. With the development of the linear ion trap, the problem arises as to how it is possible to keep ions of both polarities in a device characterized by a DC potential well in the axial dimension. Various solutions have been advanced including using an unbalance RF [54] or using a trapping AC potential $[53,55]$ on the end electrodes.

The overlap of the positive and negative ion populations can be easily studied using simulation. Figure 9a shows a SCIEX linear ion trap which consists of two pairs of round rods and one pair of flat endplates. The half distance between the opposite rods is $4.17 \mathrm{~mm}$ and the inscribed radius of each rods is $4.70 \mathrm{~mm}$. The total length of the round rods is $40 \mathrm{~mm}$. The endplates each have a $3 \mathrm{~mm}$ diameter hole in the center and are a 2-mm distance away from the ends of the rods. Ion mutual storage was achieved by applying unbalanced RF on the two pairs of round rods; such an unbalanced RF generates a time-dependent field along the $\mathrm{z}$ axis. In the simulations, 5000 ions of $\mathrm{m} / \mathrm{z} 400$ and 5000 ions of $\mathrm{m} / \mathrm{z}$ -400 were started in the center of the linear trap. A two-phase trapping RF signal, $650 \mathrm{kHz}$, was applied to the two pairs of rods. The applied RF amplitude was selected to be $308 \mathrm{~V}_{0-p}$ on the $\mathrm{x}$ pair rods and $92 \mathrm{~V}_{0-p}$ on the y pair. The two endplates were kept grounded. The simulation time was $10 \mathrm{~ms}$ and the helium buffer gas pressure was 1 mTorr. The ion-ion Coulombic interactions (space charge) cause broadening of the ion cloud and the merging of the ion frequencies. However, due to the low ion density simulated for the linear ion trap, the space charge effects are negligible and thus were not included in this simulation. Figure $9 \mathrm{~b}$ shows the ion distribution and the overlap of the positive and negative ions in $x-z$ and $y-z$ planes. Both polarities are spread out and confined inside a relatively small region with $1 \mathrm{~mm}$ in width in $\mathrm{x}$ and $\mathrm{y}$ directions and $40 \mathrm{~mm}$ along $\mathrm{z}$ direction.

\section{Conclusions}

The newest version of the ion trajectory simulation program ITSIM 6.0 allows simulations of ion motion in electrical structures with arbitrary geometries. The program CreatePot is successful as an interface program that converts the electric field output from a 3D field 

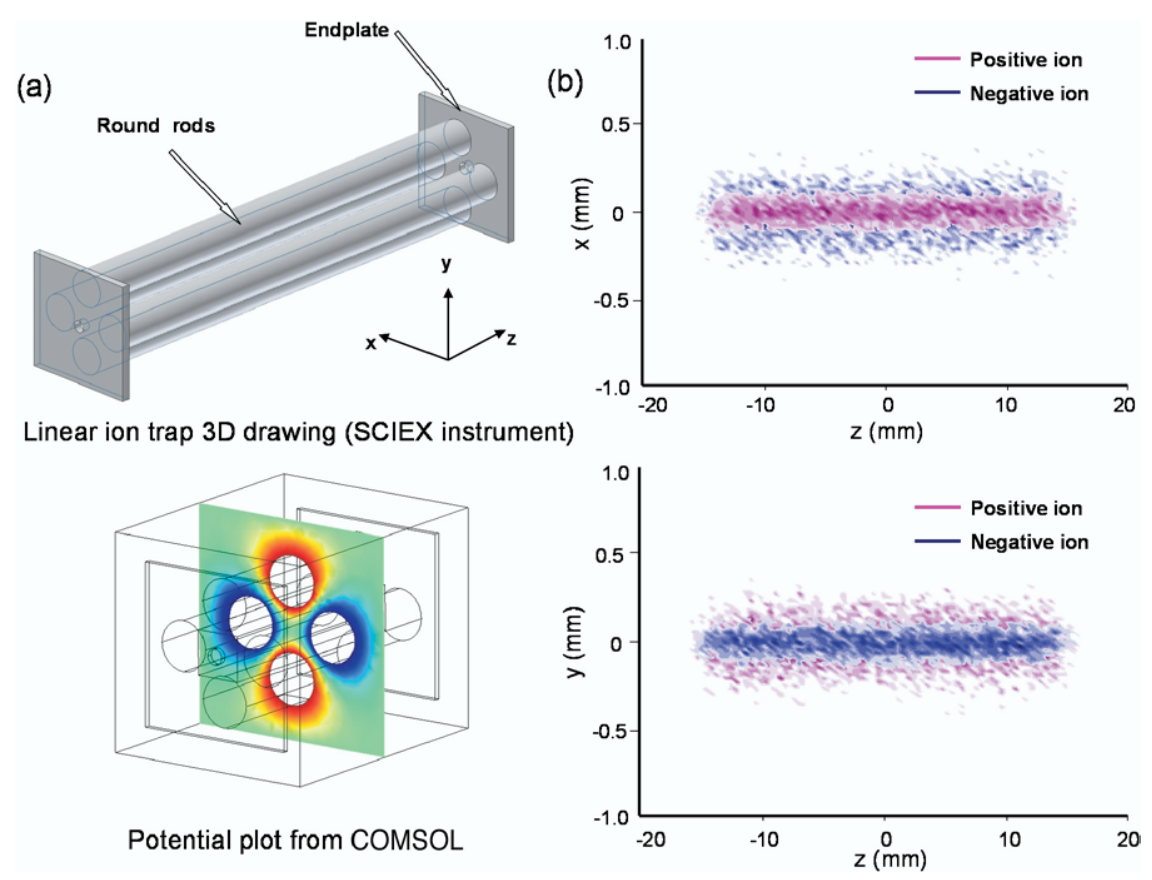

Figure 9. (a) 3D view of the SCIEX linear trap and the potential plot from COMSOL 3.0a showing the field resulting from application of two-phase RF to the two pairs of rods. (b) Ion distribution and overlap of positive and negative ions, $m / z 400$ and $m / z-400$, in x-z and y-z planes using an unbalanced RF. Two-phase RF, $650 \mathrm{kHz}$, was applied to the two pairs of rods. The RF amplitude applied to the $\mathrm{x}$ pair rods was 308 and $92 \mathrm{~V}_{0-p}$ on the y pair. The two endplates were kept at ground. The helium buffer gas pressure was 1 mTorr.

solver into a field array file readable by ITSIM 6.0. The finite element field solver COMSOL 3.0a was used in this study and the accuracy of the calculated field was studied for the ideal quadrupole ion trap for different mesh densities. Simulations for a variety of 3D devices, including ion transport systems and linear ion traps, demonstrated both qualitative and quantitative characteristics of ITSIM 6.0, elucidating ion motion behavior and predicting experimental results. The development of ITSIM will continue and new capabilities will be added in future, including simulations of ion motion in the presence of magnetic fields and gas flow. The 3D display of ion trajectories is also planned.

\section{Acknowledgments}

The authors acknowledge support from the Homeland Security Advanced Research Projects Agency (HSARPA) and the Office of Naval Research, and valuable discussions about COMSOL with Yi Wang and Yuehui Ouyang.

\section{References}

1. March, R. E. Quadrupole Ion Trap Mass Spectrometry: Theory, Simulation, Recent Developments, and Applications. Rapid Commun. Mass Spectrom. 1998, 12, 1543-1554.

2. Diaz, J. A.; Giese, C. F.; Gentry, W. R. Sub-Miniature EXB Sector-Field Mass Spectrometer. J. Am. Soc. Mass Spectrom. 2001, 12, 619-632.

3. Marinach, C.; Brunot, A.; Beaugrand, C.; Bolbach, G.; Tabet, J. C. Simulation of Ion Beam and Optimization of Orthogonal Tandem Ion Trap/Reflector Time-of-Flight Mass Spectrometry.Int. J. Mass Spectrom. 2002, 213, 45-62.

4. Dawson, P. H.; Whetten, N. R. Ion Storage in Three-Dimensional, Rotationally Symmetric, Quadrupole Fields. I. Theoretical Treatment J. Vac. Sci. Technol. 1968, 5, 91-107.
5. March, R. E.; Todd, J. F. J., Eds; Practical Aspects of Ion Trap Mass Spectrometry, Vol. I: Fundamentals of Ion Trap Mass Spectrometry; CRC Press: Boca Raton, FL, 1995.

6. Xiang, X.; Guan, S.; Marshall, A. G. Simulated Ion Trajectory and Induced Signal in Ion Cyclotron Resonance Ion Traps. J. Am. Soc. Mass Spectrom. 1994, 5, 238-249.

7. Mitchell, D. W. Realistic Simulation of the Ion Cyclotron Resonance Mass Spectrometer Using a Distributed Three-Dimensional Particle-inCell Code. J. Am. Soc. Mass Spectrom. 1999, 10, 136-152.

8. Franzen, J. Simulation Study of an Ion Cage with Superimposed Multipole Fields. Int. J. Mass Spectrom. Ion Processes 1991, 106, 63-78.

9. Vedel, F.; Andre, J.; Vedel, M.; Brincourt, G. Computed Energy and Spatial Statistical Properties of Stored Ions Cooled by a Buffer Gas. Phys. Rev. A 1983, 27, 2321-2330.

10. Vedel, F.; Andre, J. Influence of Space Charge on the Computed Statistical Properties of Stored Ions Cooled by a Buffer Gas in a Quadrupole RF Trap. Phys. Rev. A 1984, 29, 2098-2101.

11. Londry, F. A.; Alfred, R. L.; March, R. E. Computer Simulation of Single-Ion Trajectories in Paul-Type Ion Traps. Int. J. Mass Spectrom. 1993, 4, 687-705.

12. Sevugarajan, S.; Menon, A. G. Frequency Perturbation in Nonlinear Paul Traps: A Simulation Study of the Effect of Geometric Aberration, Space Charge, Dipolar Excitation, and Damping on Ion Axial Secular Frequency. Int. J. Mass Spectrom. 2000, 197, 263-278.

13. Blain, M. G.; Riter, L. S.; Cruz, D.; Austin, D. E.; Wu, G.; Plass, W. R.; Cooks, R. G. Towards the Hand-Held Mass Spectrometer: Design Considerations, Simulation, and Fabrication of Micrometer-Scaled Cylindrical Ion Traps. Int. J. Mass Spectrom. 2004, 236, 91-104.

14. Ding, L.; Sudakov, M.; Kumashiro, S. A Simulation Study of the Digital Ion Trap Mass Spectrometer. Int. J. Mass Spectrom. 2002, 221, 117-138.

15. Lammert, S. A.; Plass, W. R.; Thompson, C. V.; Wise, M. B. Design, Optimization, and Initial Performance of a Toroidal RF Ion Trap Mass Spectrometer. Int. J. Mass Spectrom. 2001, 212, 25-40.

16. Wu, G.; Cooks, R. G.; Ouyang, Z. Geometry Optimization for the Cylindrical Ion Trap: Field Calculations, Simulations, and Experiments. Int. J. Mass Spectrom. 2005, 241, 119-132.

17. Dahl, D. A. SIMION for the Personal Computer in Reflection. Int. J. Mass Spectrom. 2000, 200, 3-25.

18. Veryovkin, I. V.; Calaway, W. F.; Pellin, M. J. A Virtual Reality Instrument: Near-Future Perspective of Computer Simulations of Ion Optics. Nucl. Instrum. Methods A 2004, 519, 363-372.

19. Forbes, M. W.; Sharifi, M.; Croley, T.; Lausevic, Z.; March, R. E. Simulation of Ion Trajectories in a Quadrupole Ion Trap: A Comparison of Three Simulation Programs. J. Mass Spectrom. 1999, 34, 1219-1239.

20. March, R. E.; Londry, F. A.; Alfred, R. L.; Todd, J. F. J.; Penman, A. D.; Vedel, F.; Vedel, M. Resonance Excitation of Ions Stored in a Quadru- 
pole Ion Trap. Part III. Introduction to the Field Interpolation Simulation Method. Int. J. Mass Spectrom. Ion Processes 1991, 110, 159-178.

21. March, R. E.; Todd, J. F. J., Eds. Quadrupole Ion Trap Mass Spectrometry; John Wiley and Sons, Inc.: Hoboken, NJ, 2005, Chap IV.

22. Bui, H. A.; Cooks, R. G. Windows Version of the Ion Trap Simulation Program ITSIM: A Powerful Heuristic and Predictive Tool in Ion Trap Mass Spectrometry. J. Mass Spectrom. 1998, 33, 297-304.

23. Plass, W. R.; Ph.D. Thesis, Justus Liebig Universität Giessen, Giessen, Germany, 2001

24. Londry, F. A.; Hager, J. W. Mass Selective Axial Ion Ejection from a Linear Quadrupole Ion Trap. J. Am. Soc. Mass Spectrom. 2003, 14, $1130-1147$.

25. Hieke, A. GEMIOS-a 64-Bit Multi-Physics Gas and Electromagnetic Ion Optical Simulator. Proceedings of the 51st ASMS Conference on Mass Spectrometry and Allied Topics; Montreal, Canada, June 2003.

26. Hieke, A. 3D Electro-Pneumatic Monte-Carlo Simulations of Ion Trajectories and Temperatures during RF quadrupole injection in the presence of gas flow fields. Proceedings of the 52nd ASMS Conference on Mass Spectrometry and Allied Topics; Nashville, TN, May 2004

27. Weil, C.; Nappi, M.; Cleven, C. D.; Wollnik, H.; Cooks, R. G. Multiparticle Simulation of Ion Injection into the Quadrupole Ion Trap Under the Influence of Helium Buffer Gas Using Short Injection Times and DC Pulse Potential. Int. J. Mass Spectrom. 1996, 10, 742-750.

28. Julian, R. K.; Reiser, H.-P.; Cooks, R. G. Large Scale Simulation of Mass Spectra Recorded with a Quadrupole Ion Trap Mass Spectrometer. Int. J. Mass Spectrom. 1993, 123, 85-96.

29. Reiser, H.-P.; Julian, R. K.; Cooks, R. G. A Versatile Method of Simulation of the Operation of Ion Trap Mass Spectrometers. Int. J. Mass Spectrom. 1992, 121, 49-63.

30. Plass, W. R.; Cooks, R. G. A Model for Energy Transfer in Inelastic Molecular Collisions Applicable at Steady State or Non-Steady State and for an Arbitrary Distribution of Collision Energies. J. Am. Soc. Mass Spectrom. 2003, 14, 1348-1359.

31. Wells, M. J.; Plass, W. R.; Cooks, R. G. Control of Chemical Mass Shifts in the Quadrupole Ion Trap through Selection of Resonance Ejection Working Point and RF Scan Direction. Anal. Chem. 2000, 72, 2677-2683.

32. Wells, M. J.; Plass, W. R.; Patterson, G. E.; Ouyang, Z.; Badman, E. R.; Cooks, R. G. Chemical Mass Shifts in Ion Trap Mass Spectrometry: Experiments and Simulations. Anal. Chem. 1999, 71, 3405-3415.

33. Plass, W. R.; Li, H.; Cooks, R. G. Theory, Simulation, and Measurement of Chemical Mass Shifts in RF Quadrupole Ion Traps. Int. J. Mass Spectrom. 2003, 228, 237-267.

34. Badman, E. R.; Johnson, R. C.; Plass, W. R.; Cooks, R. G. A Miniature Cylindrical Quadrupole Ion Trap: Simulation and Experiment. Anal. Chem. 1998, 70, 4896-4901.

35. Wells, J. M.; Badman, E. R.; Cooks, R. G. A Quadrupole Ion Trap with Cylindrical Geometry Operated in the Mass-Selective Instability Mode. Anal. Chem. 1998, 70, 438-444.

36. Jackson, G. P.; Hyland, J. J.; Laskay, U. A. Energetics and Efficiencies of Collision-Induced Dissociation Achieved During the Mass Acquisition Scan in a Quadrupole Ion Trap. Rapid Commun. Mass Spectrom. 2006, 19, 3555-3563.

37. Dobson, G.; Murrell, J.; Despeyroux, D.; Wind, F.; Tabet, J. C. Investigation into Factors Affecting Precision in Ion Trap Mass Spectrometry Using Different Scan Directions and Axial Modulation Potential Amplitudes. J. Mass Spectrom. 2004, 39, 1295-1304.
38. Dobson, G.; Murrell, J.; Despeyroux, D.; Wind, F.; Tabet, J. C. Influence on Mass-Selective Ion Ejection of the Phase Difference Between the Driven RF and the Axial Modulation Potentials. J. Mass Spectrom. 2005, $40,714-721$.

39. Chaudhary, A.; van Amerom, F. H. W.; Short, R. T.; Bhansali, S. Fabrication and Testing of a Miniature Cylindrical Ion Trap Mass Spectrometer Constructed from Low Temperature Cofired Ceramics. Int. J. Mass Spectrom. 2006, 251, 32-39.

40. Plass, W. R. Theory of Dipolar DC Excitation and DC Tomography in the RF Quadrupole Ion Trap. Int. J. Mass Spectrom. 2000, 202, 175-197.

41. Hu, Q.; Noll, R. J.; Wu, G.; Makarov, A.; Plass, W. R.; Cooks, R. G. Ion Motion Control in the Orbitrap Mass Analyzer. Proceedings of the 53rd ASMS Conference on Mass Spectrometry and Allied Topics; San Antonio, TX, June 2005.

42. Schwartz, J. C.; Senko, M. W.; Syka, J. E. P. A Two-Dimensional Quadrupole Ion Trap Mass Spectrometer. J. Am. Soc. Mass Spectrom. 2002, 13, 659-669.

43. Hager, J. M. A New Linear Ion Trap Mass Spectrometer. Rapid Commun. Mass Spectrom. 2002, 16, 512-526.

44. Ouyang, Z.; Wu, G.; Song, Y.; Li, H.; Plass, W. R.; Cooks, R. G. Rectilinear Ion Trap: Concepts, Calculations, and Analytical Performance of a New Mass Analyzer. Anal. Chem. 2004, 76, 4595-4605.

45. Steer, M. B.; Bandler, J. W.; Snowden, C. M. Computer-Aided Design of $\mathrm{RF}$ and Microwave Circuits and Systems. IEEE Trans. Microwave Theory Tech. 2002, 50, 996-1005.

46. Billen, J. H.; Young, L. M. Poisson/Superfish on PC Compatibles. Proceedings of the 1993 Particle Accelerator Conference, Washington, D.C., May 1993, pp 790-792.

47. Hagg, C.; Szabo, I. New Ion-Optical Devices Utilizing Oscillatory Electric Fields. IV. Computer Simulations of the Transport of an Ion Beam Through an Ideal Quadrupole, Hexapole, and Octopole Operating in the RF-Only Mode. Int. J. Mass Spectrom. Ion Proc. 1986, 73, 295-312.

48. Song, Q.; Kothari, S.; Senko, M. A.; Schwartz, J. C.; Amy, R. J. W.; Stafford, G. C.; Cooks, R. G.; Ouyang, Z. Rectilinear Ion Trap Mass Spectrometers with Atmospheric Pressure Interface and Electrospray Ionization Source. Anal. Chem. 2006, 78, 718-725.

49. Zhang, C.; Chen, H.; Guymon, A. J.; Wu, G.; Cooks, R. G.; Ouyang, Z. Instrumentation and Methods for Ion and Reaction Monitoring Using A Non-Scanning Rectilinear Ion Trap. Int. J. Mass Spectrom., in press.

50. Levine, R. D., Ed. Molecular Reaction Dynamics; Cambridge University Press: New York, NY, 2005, pp 356-393.

51. Baer, T.; Dutuit, O.; Mestdagh, H.; Rolando, C. Dissociation Dynamics of n-Butylbenzene Ions: The Competitive Production of $\mathrm{m} / \mathrm{z} 91$ and 92 Fragment Ions. J. Phys. Chem. 1988, 92, 5674-5679.

52. Gunawardena, H. P.; He, M.; Chrisman, P. A.; Pitteri, S. J.; Hogan, J. M.; Hodges, B. D. M.; McLuckey, S. A. Electron Transfer versus Proton Transfer in Gas-Phase Ion/Ion Reactions of Polyprotonated Peptides. J. Am. Chem. Soc. 2005, 127, 12627-12639.

53. Syka, J. E. P.; Coon, J. J.; Schroeder, M. J.; Shabanowitz, J.; Hunt, D. F. Peptide and Protein Sequence Analysis by Electron Transfer Dissociation Mass Spectrometry. Proc. Natl. Acad. Sci. U.S.A. 2004, 101, $9528-$ 9533.

54. Xia, Y.; Wu, J.; McLuckey, S. A.; Londry, F. A.; Hager, J. W. Mutual Storage Mode Ion/Ion Reactions in a Hybrid Linear Ion Trap. J. Am. Soc. Mass Spectrom. 2005, 16, 71-81.

55. Xia, Y.; Liang, X.; McLuckey, S. A. Sonic Spray as a Dual Polarity Ion Source for Ion/Ion Reaction. Anal. Chem. 2005, 77, 3683-3689. 